\title{
Active Turbine Tip Clearance Control Trade Space Analysis of an Advanced Geared Turbofan Engine
}

\author{
Jonathan Kratz (NASA GRC) \\ Jeffryes Chapman (NASA GRC) \\ AIAA Propulsion \& Energy Forum \\ 11 July 2018
}




\section{Outline}

Summary

- Illustration of a systematic approach for modeling high pressure turbine tip clearance

- Application is to a conceptual $\mathrm{N}+3$ geared turbofan with a compact gas turbine (CGT)

- Presentation of a sensitivity study and a parametric study of actuators that provides preliminary requirement for implementing active turbine tip clearance control

Outline

- Background/Motivation

- Modeling

- Control Approach

- Sensitivity Studies

- Parametric Actuator Studies

- Conclusions 


\section{Background}

- Tip clearance, especially in the high pressure turbine, can lead to inefficiencies

- Poor sealing can lead to flow leakages that:

- Reduce work extraction and thus efficiency

- Reduce flow capacity

- Produces tip vortices that can lead to increased noise

- Makes the turbine work harder to spin the shaft $\rightarrow$ requires more fuel and higher operating temperatures

- For a large gas turbine:

- 10 mil of clearance $\rightarrow 1 \%$ difference in turbine efficiency \& $10{ }^{\circ} \mathrm{C}$ in exhaust gas temperature (impacts time on wing)

Image is from: Lattime, S.B., and Steinetz, B.M., "Turbine engine clearance control systems: current practices and future directions," American Control Conference, 2001.

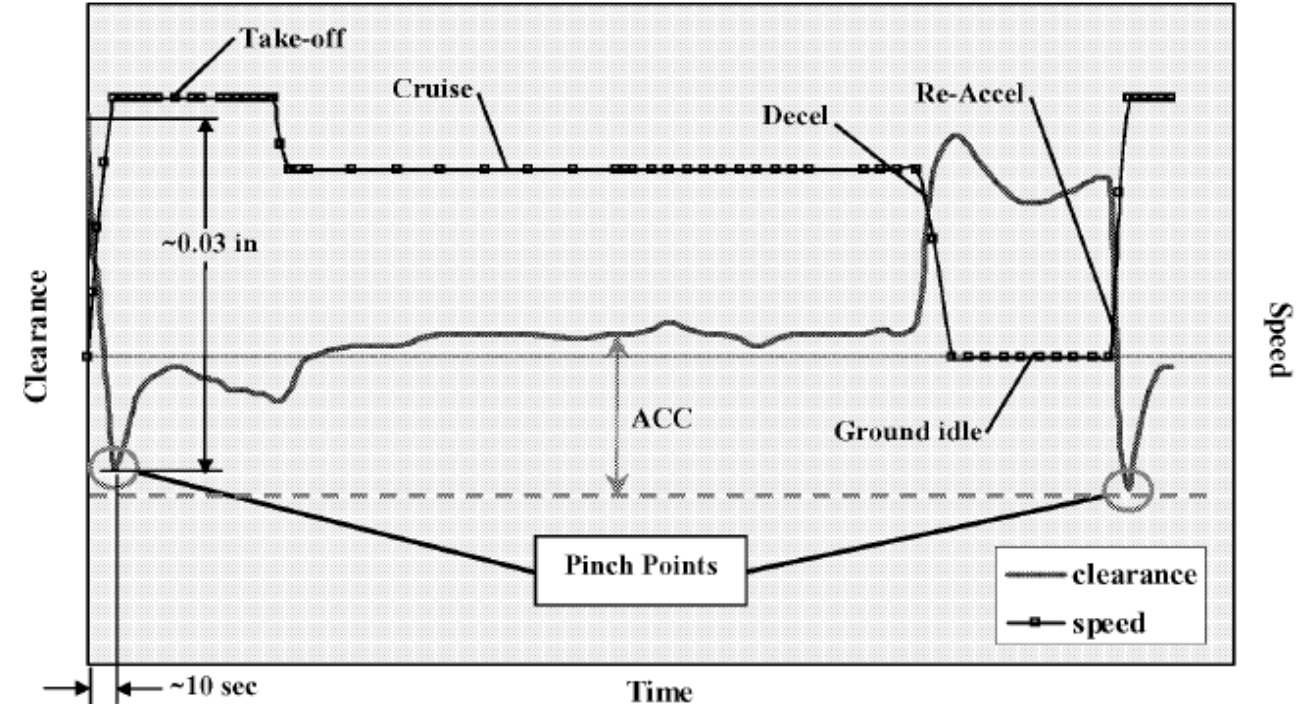

- Performance sensitivity to turbine tip clearance is expected to be greater for CGTs

- Tip clearance gap varies through the course of a flight and is impacted by:

- Degradation

- Mechanical loads

- Thermal loads 


\section{Background}

- Current approach to turbine tip clearance control

- Cooling air is blown over the casing around the turbine to contract the casing and close down the clearance, particularly during cruise

- Can only actively effect the tip clearance by closing it

- Scheduled control logic

- No direct tip clearance measurement and limited feedback

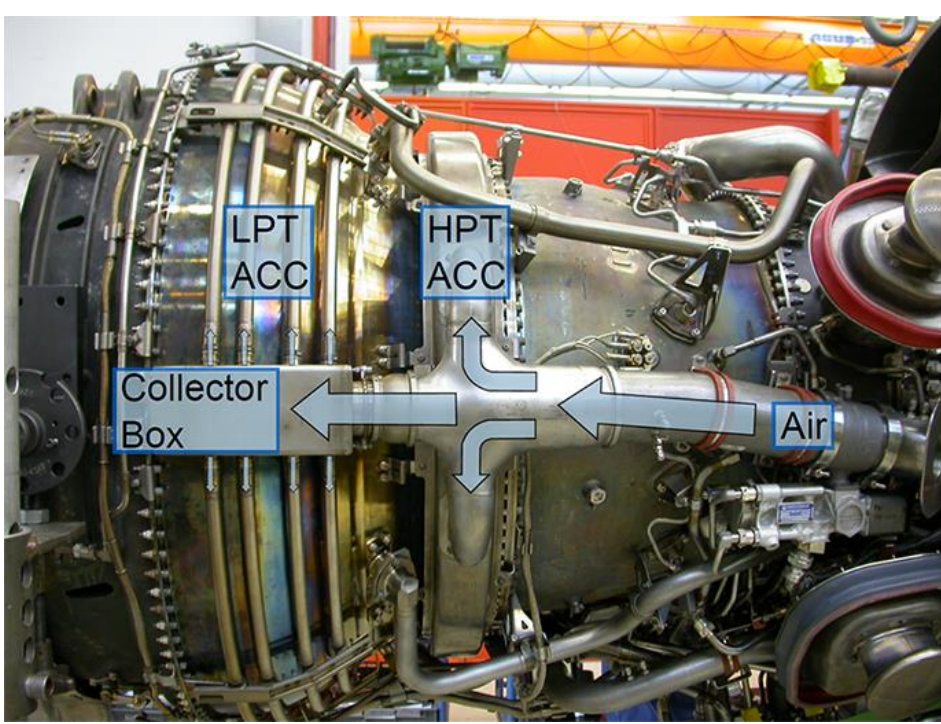

- Proposed approach - Higher Bandwidth - Active Turbine Tip Clearance Control

- Could be a variety of actuator types and system designs

- Use of an actively controlled "faster" actuation system to maintain tighter clearance

- Can effect tip clearance by both opening and closing the gap

- Utilizes a direct tip clearance measurement or reliable estimator

Image is from: Soghe, R.D., Andreini, A. "Numerical Characterization of Pressure Drop Across the Manifold of Turbine Casing Cooling System", ASME Journal of Turbomachinery, Vol. 135, Issue 3 (2013) 


\section{Modeling}

- System level physics-based approach

- Considers axisymmetric tip clearance variations due to centrifugal and thermal loads

- Neglects asymmetric variations that are much less significant

- Models the following components:

- Blade

- Rotor Disc

- Shroud/Case

- Thermal expansion calculations use a 1-D linear elastic expansion model that utilizes the average temperature of the component

- Rotor Disc \& Shroud/Case - 1-D finite difference method

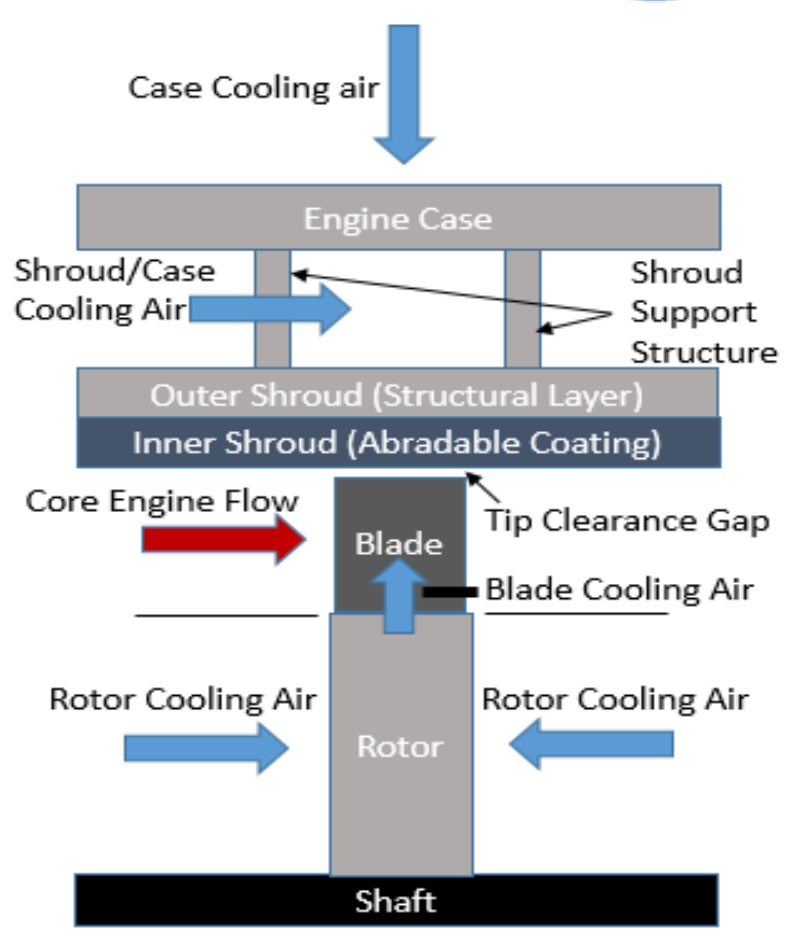

- Blade-Lumped capacitance model

- Centrifugal expansion of the rotor disc \& blade

- Modeled with algebraic equations under assumptions of simplified geometries

- Temperature dependent thermal \& mechanical properties are considered

- A MATLAB/Simulink Library, the Tip Clearance Modeling Library (TCML) was created to implement this approach in parameterized manner

- Download available at https://github.com/nasa/TCML 


\section{Control Approach}

- Baseline control approach

- Implements cooling on the outer engine casing using bleed air from the bypass duct

- A bleed schedule was designed to maintain a prescribed tip clearance that provide acceptable margin for transients

- The schedule utilizes the existing sensor suite
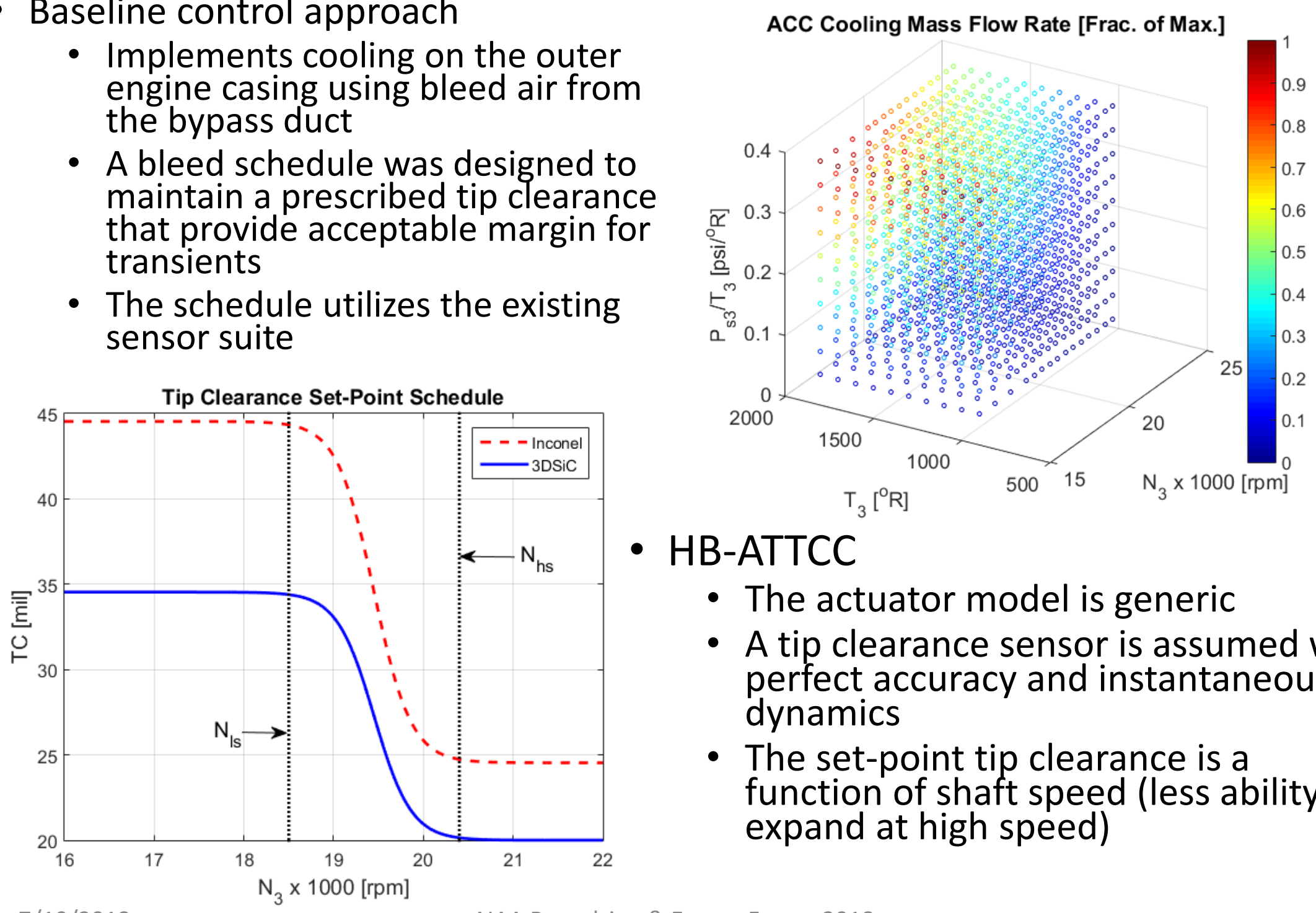

- HB-ATTCC

- The actuator model is generic

- A tip clearance sensor is assumed with perfect accuracy and instantaneous dynamics

- The set-point tip clearance is a function of shaft speed (less ability to expand at high speed) 


\section{Modeling}

- The tip clearance model was integrated with the AGTF30 engine model

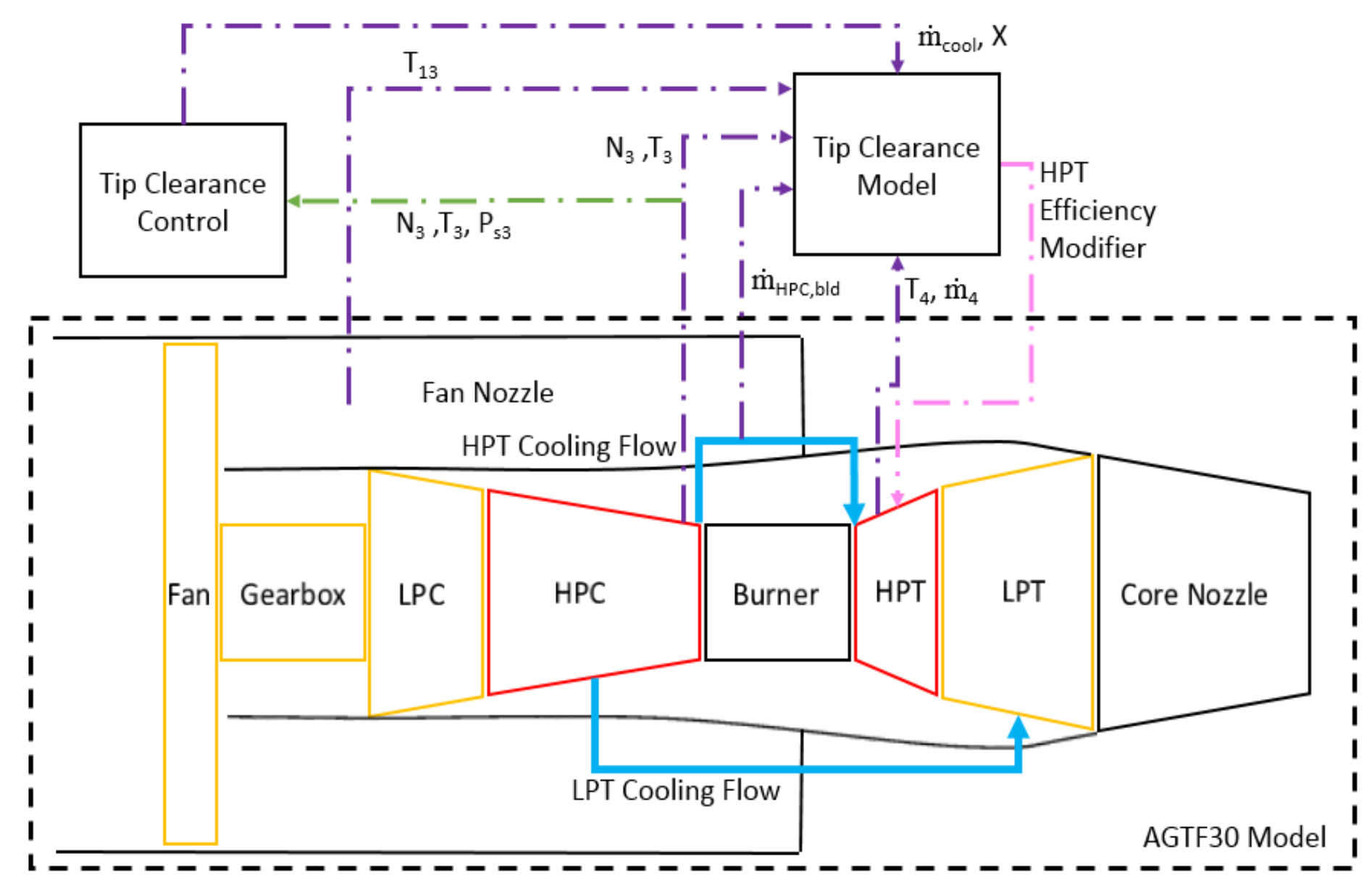




\section{Baseline Models}
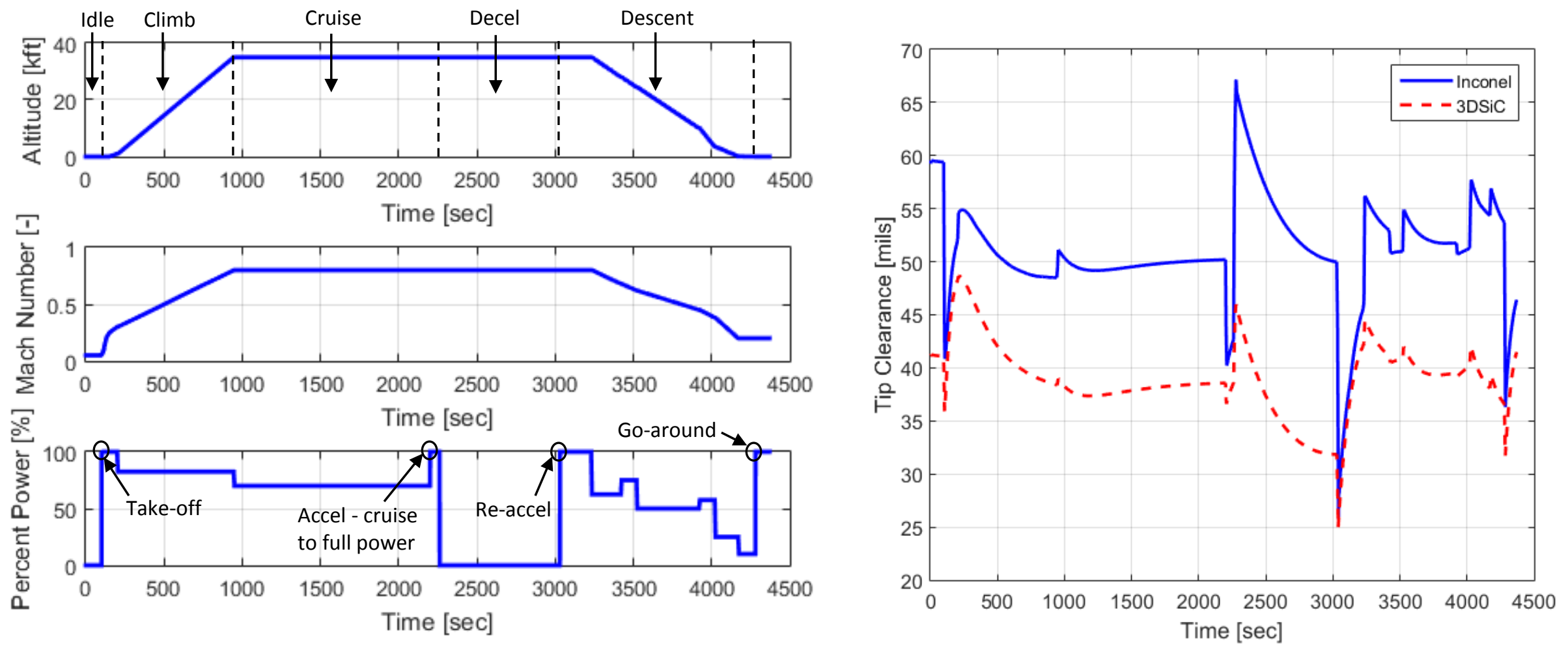

- 2 different turbine configurations were considered, distinguished by the blade material

- Inconel

- 3-D Angle Interlock SiC/SiC Hybrid Matrix Ceramic Matrix Composite (3DSiC)

- Flight profile contains various potential worst case scenarios

- The "stationary cold" tip clearance was adjusted in each case to enforce a minimum tip clearance of 25 mil (accounts for axisymmetric variation and other uncertainties/conservativeness that comes with the traditional control approach) 


\section{Sensitivity Studies}
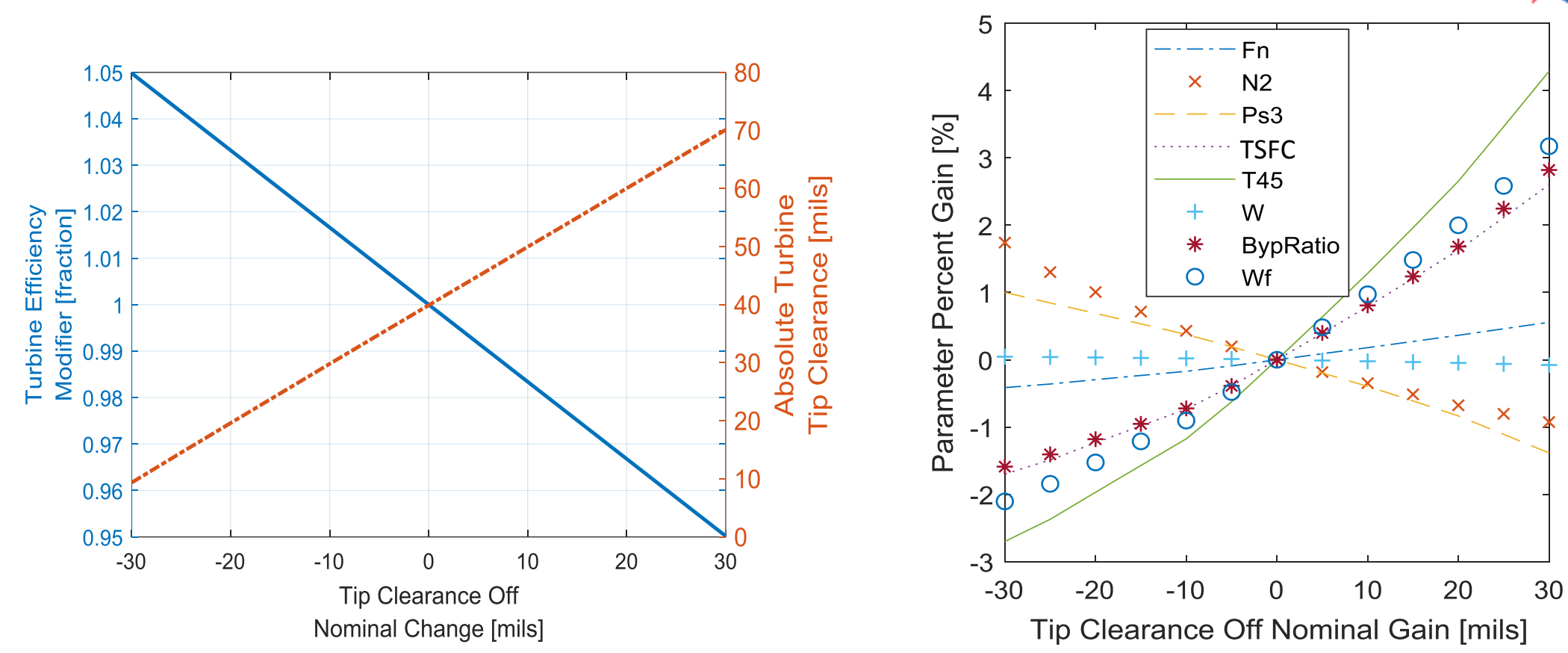

- AGTF30 was run at the steady-state cruise condition and various tip clearances were enforced

- $\sim 5 \%$ change in turbine efficiency for a 30 mil variation in tip clearance $(\sim 1.67 \%$ difference for every $10 \mathrm{mil}$ )

- Inter-turbine temperature $\left(T_{45}\right)$, fuel flow rate (Wf), and thrust specific fuel consumption (TSFC) are the most impacted parameters 


\section{Sensitivity Studies}

\begin{tabular}{|l|c|c|c|}
\hline Material Parameter & Inconel 718 & 3DSiC & Units \\
\hline Density & 15.89 & 4.7725 & slug/ $/ \mathrm{ft}^{3}$ \\
\hline Thermal expansion coefficient & $1.05 \times 10^{-5}$ & $0.144 \times 10^{-5}$ & $1 /{ }^{\circ} \mathrm{R}$ \\
\hline Poisson's ratio & 0.4093 & 0.17 & $\mathrm{~N} / \mathrm{A}$ \\
\hline Specific heat & 5.47 & 8.73 & $\mathrm{Btu} /\left(\mathrm{slug}-{ }^{\circ} \mathrm{R}\right)$ \\
\hline Modulus of elasticity & $1.19 \times 10^{9}$ & $3.17 \times 10^{9}$ & $\mathrm{Ib}_{\mathrm{f}} / \mathrm{ft}^{2}$ \\
\hline
\end{tabular}

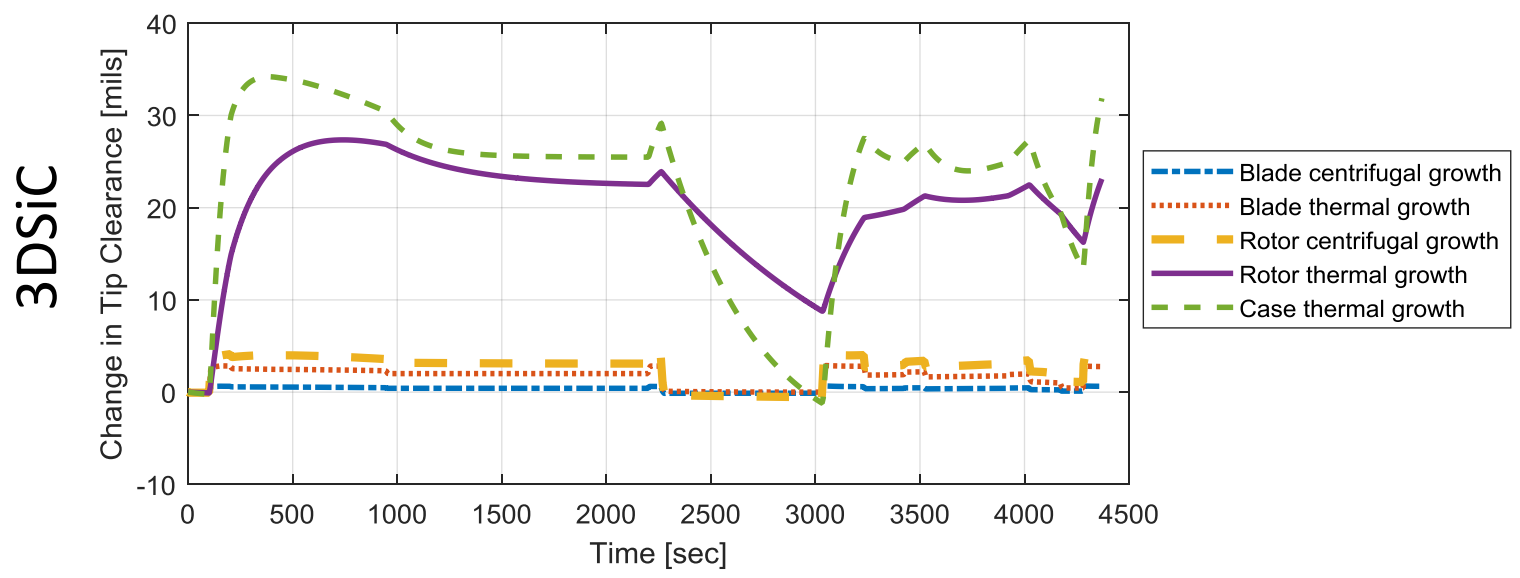

- 3DSiC blades lower

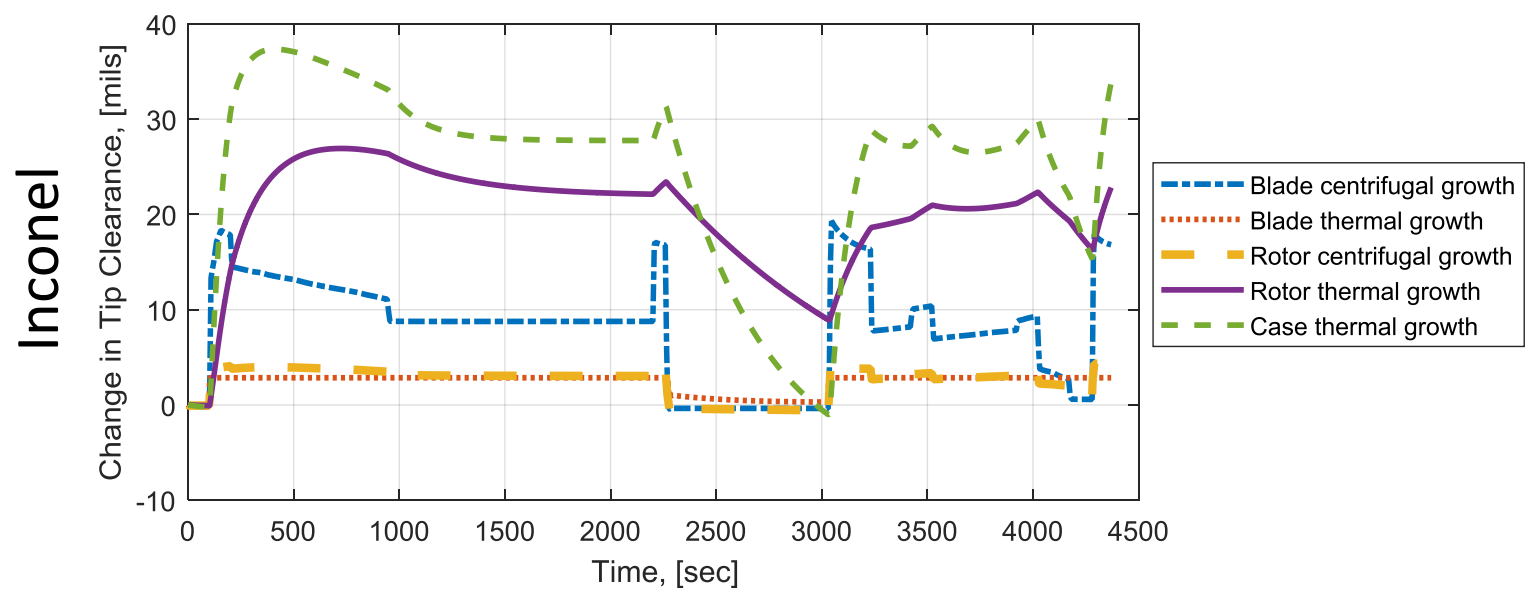
density and higher modulus of elasticity reduces its expansion considerably

- However, it was found that the cooling schedule requires $\sim 50 \%$ more bleed than the Inconel blade when paired with the Inconel casing 


\section{Parametric Actuator Studies}

- Evaluated bandwidth (BW), rate limit ( $R L)$, range (R), and deadband (DB) needs

- Took a look at weight and force implication

- Simulations utilized the flight profile below (R \& DB) or an accel from the cruise point to full throttle (BW \& RL studies)

- For each set of actuator parameters, the tip clearance set-point schedule was adjusted such that the minimum tip clearance observed in the simulation was $\sim 15 \mathrm{mil}(0.09 \%$ of the blade span)

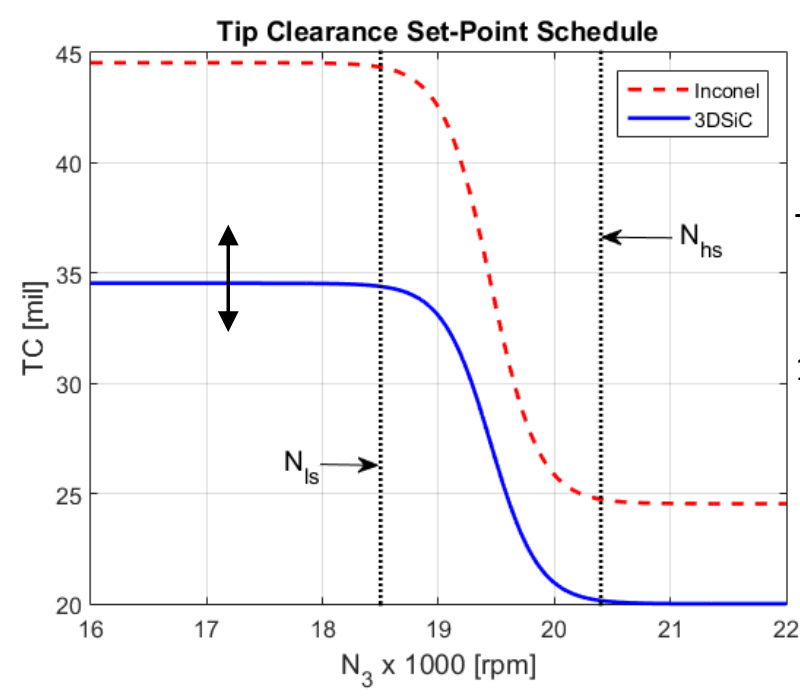

TC response $15 \mathrm{mi}$
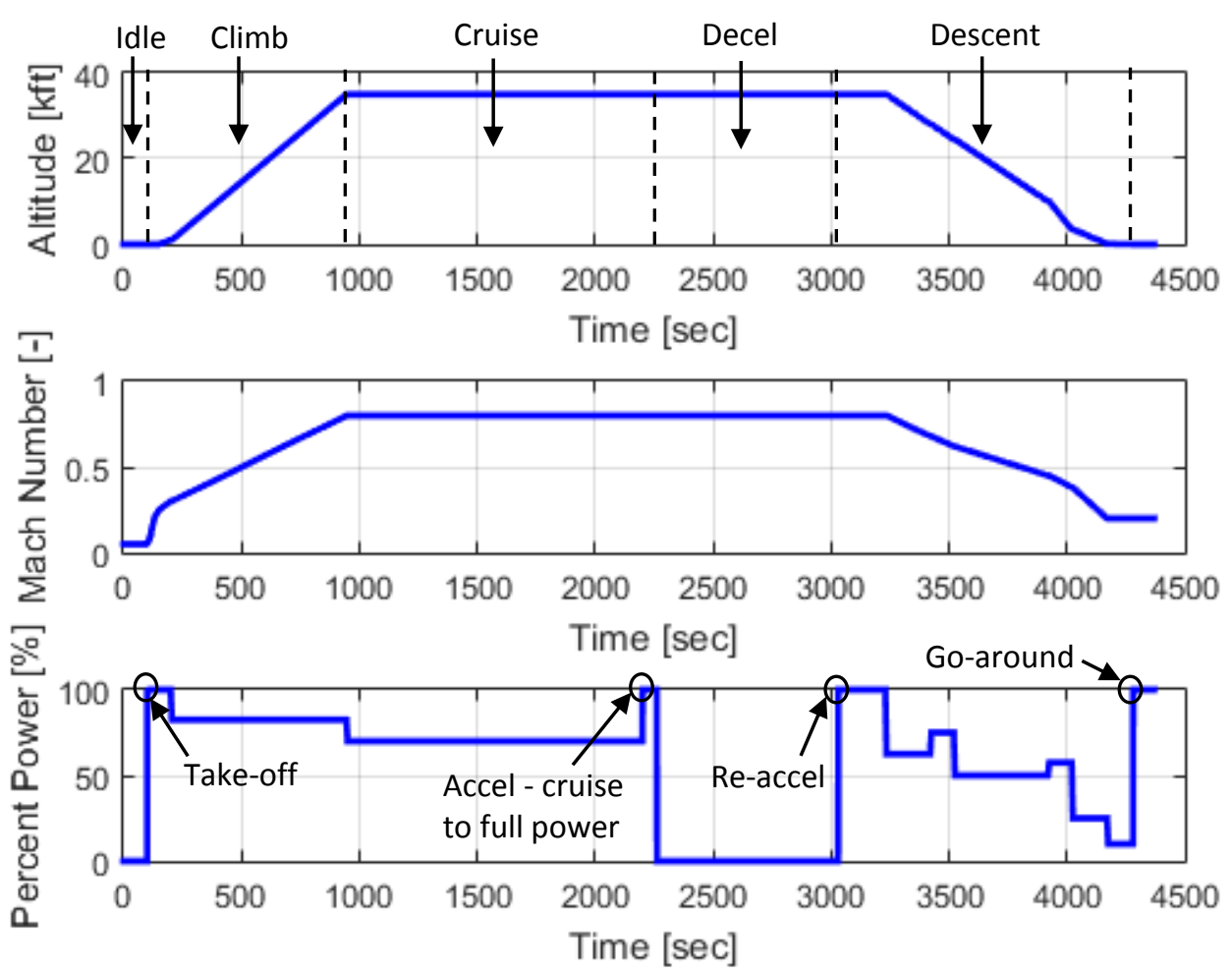


\section{Parametric Actuator Studies}
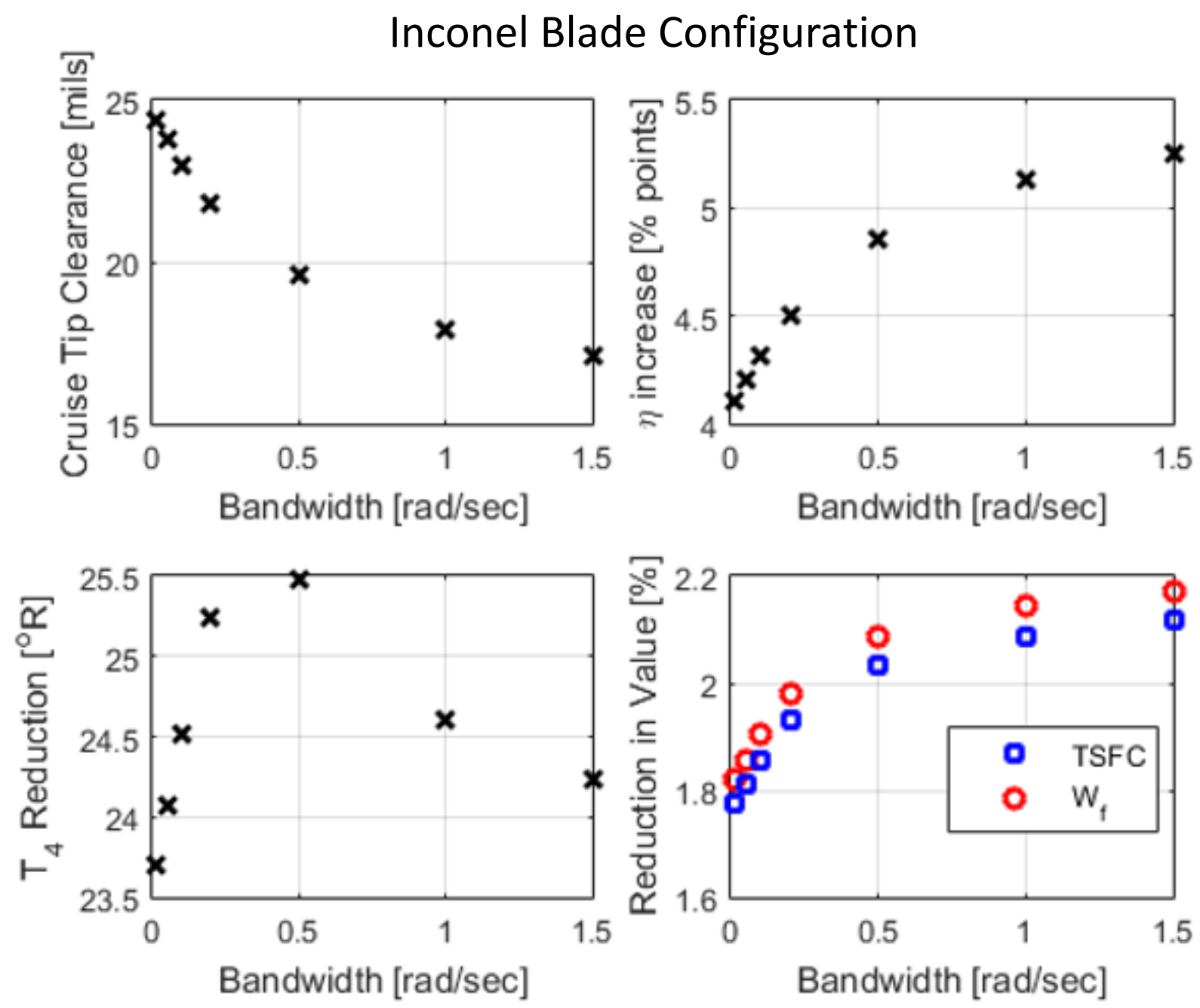

- Large benefit just from being able to actively control

- Relevant bandwidth range:

- Inconel: 0.01 $1.5 \mathrm{rad} / \mathrm{sec}$

- 3DSiC: $0.01-2$ $\mathrm{rad} / \mathrm{sec}$ 


\section{Parametric Actuator Studies}

Inconel Blade Configuration
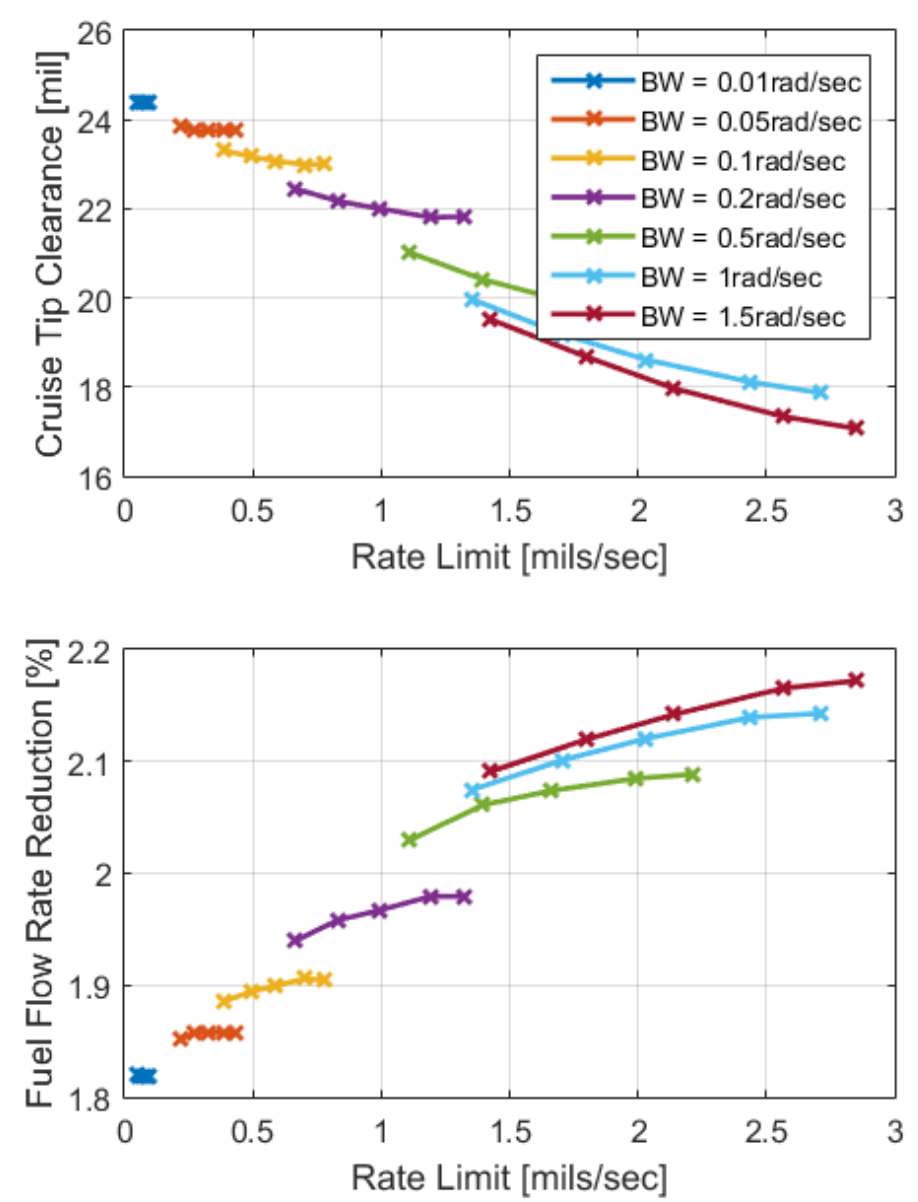
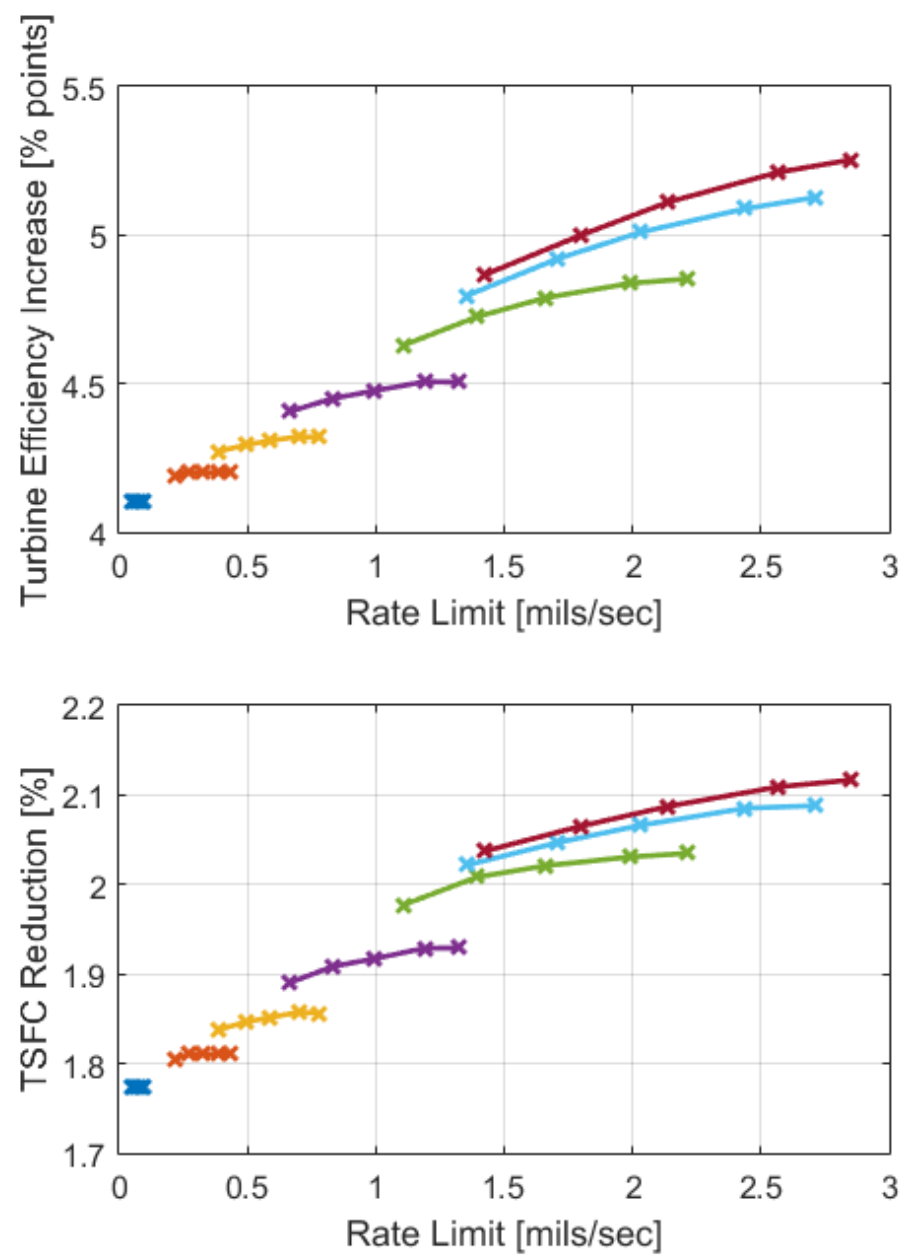

- Rate limit did not have much impact for the 3DSiC blade configuration

- The entire range of tested rate limits appears to be applicable 


\section{Parametric Actuator Studies}
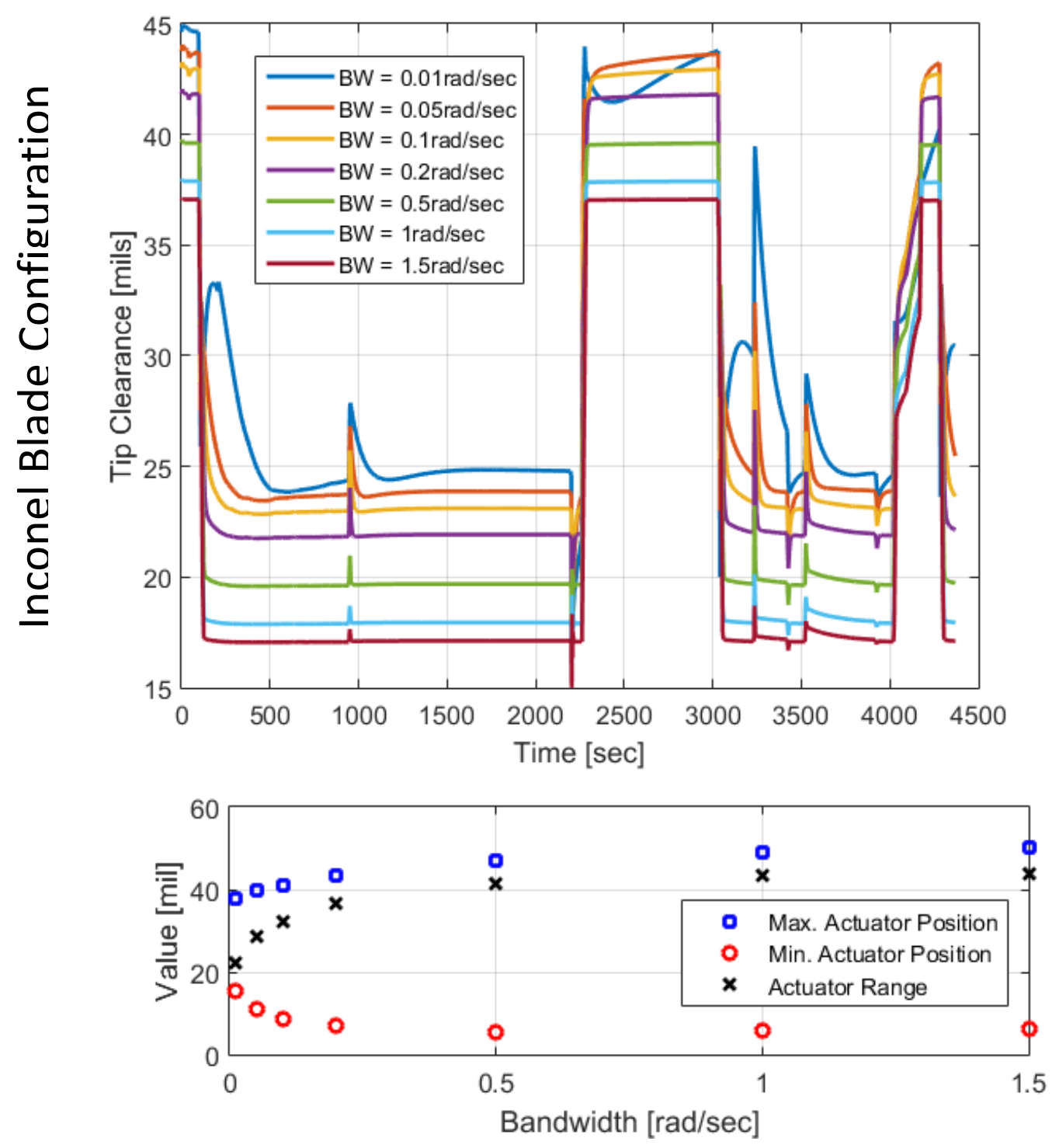

- Range: 45mils (Inconel), 30mils (3DSiC)

- For $\mathrm{BW}=0.01 \mathrm{rad} / \mathrm{sec}$ the tip clearance reduces during the deceleration period potential risk for rubbing during a subsequent acceleration

- Re-evaluate speed requirements: $\mathrm{BW} \geq$ $0.05 \mathrm{rad} / \mathrm{sec} \& \mathrm{RL} \geq$ $0.45 \mathrm{mil} / \mathrm{sec}$ 


\section{Parametric Actuator Studies}

- Solved the Breguet range equation to determine the amount of weight that could be added to the engine system on account of the actuation system for various flight ranges and fuel savings benefits

Inconel Blade Configuration
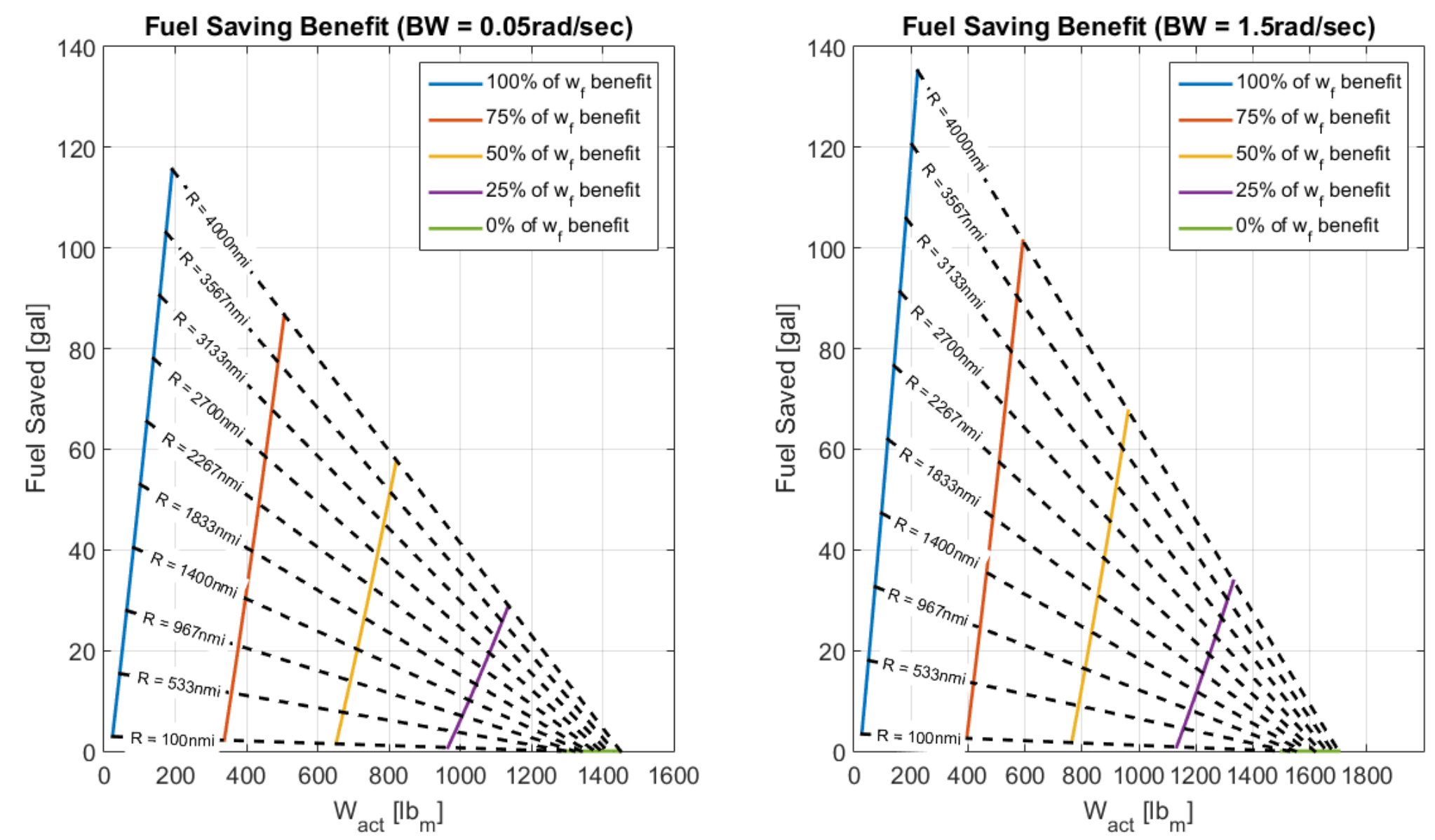


\section{Conclusions}

- HB-ATTCC would be advantageous for both of the evaluated configurations

- Significant efficiency benefits could be enabled through implementing active control to both open and close the tip clearance and does NOT necessary need to be "fast"

- Only needs to be fast enough to retreat to its set-point such that a re-accel pinch point will not violate the acceptable tip clearance margin

- Use of the 3DSiC (CMC) blade reduced the tip clearance variation but with traditional control methods required significantly more bleed ( $\sim 50 \%$ more) to cool and contract the casing

- Observations from the sensitivity analysis:

- Turbine efficiency changes by $\sim 1.67 \%$ \& fuel consumption by $\sim 0.67 \%$ for every 10 mil of tip clearance

- Largest impact is on turbine operating temperatures, fuel flow and TSFC

- Young's modulus \& density of the blade play a significant role in the natural tip clearance variation

- Effectiveness of cooling flow is limited and has diminishing effects as more flow is bled for cooling

- Recommendations based on the parametric actuator study:

- Bandwidth: Inconel - 0.05-1.5 rad/sec, 3DSiC $-0.05-2 \mathrm{rad} / \mathrm{sec}$

- Rate Limit: Inconel $-0.45 \mathrm{mil} / \mathrm{sec}, 3 \mathrm{DSiC}-0.45 \mathrm{mil} / \mathrm{sec}$

- Range: Inconel - 45-50 mil (2.5-2.8\% span), 3DSiC - 30-50 mil (1.7-2.8\% span)

- Deadband: Inconel $-<2$ mil, 3DSiC $-<0.5$ mil

- Other observations:

- Can increase $\eta$ by $3.5-5.5 \%$, reduce $T_{4}$ by $10-25^{\circ} \mathrm{R}$, and reduce TSFC by $1.25-2.2 \%$

- Can save 10-120 gal of fuel per flight compared to the baseline engine 


\section{Acknowledgements}

- This work supports the objectives and goals of NASA's Advanced Air Transportation Technology (AATT) Project funded by the Aeronautics Research Mission Directorate (ARMD).

- NASA civil servants \& contractors who contributed in some way to this effort: Sanjay Garg, Joe Grady, Ram Bhatt, Jerry Lang, Vikram Shyam, Paht Juangphanich, Dennis Culley, Joe Saus 


\section{Questions?}

Contact Information:

- Jonathan Kratz - jonathan.kratz@nasa.gov

- Jeffryes Chapman - jeffryes.w.chapman@nasa.gov TCML Link: https://github.com/nasa/TCML 


\section{EXTRA SLIDES}




\section{The Engine}

Advanced Geared Turbofan

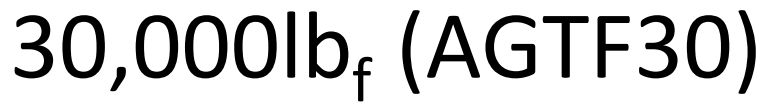

- Developed using the Toolbox for Modeling and Analysis of

Thermodynamic Systems (T-MATS)

- Based on the NASA N+3 NPSS reference engine

- Features a CGT and a variable area fan nozzle

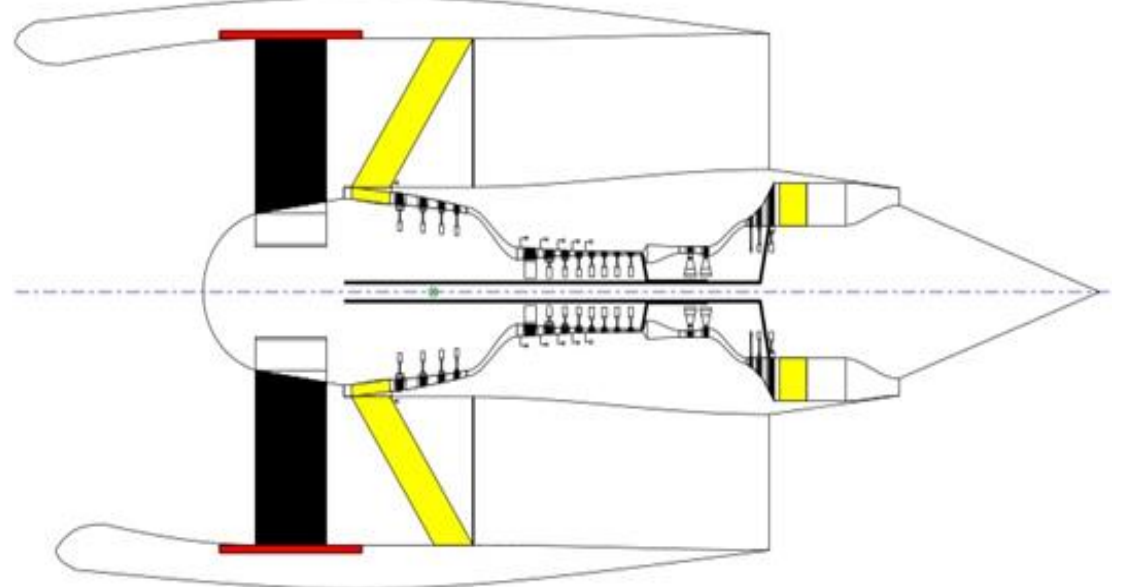

- Capable of producing $30,000 \mathrm{lb}_{\mathrm{f}}$ of thrust at the sea-level static condition

- Includes a realistically performing full-flight envelop controller 


\section{Parametric Actuator Studies}

Inconel Blade Configuration
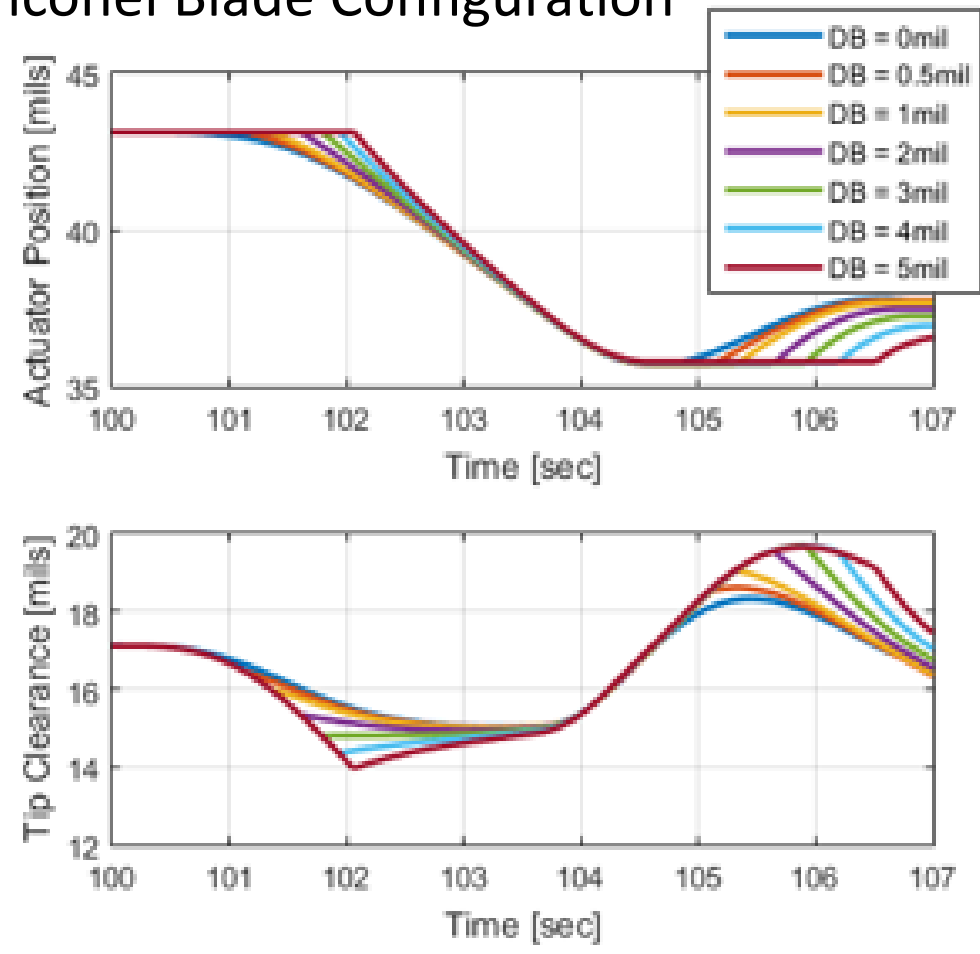

3DSiC Blade Configuration
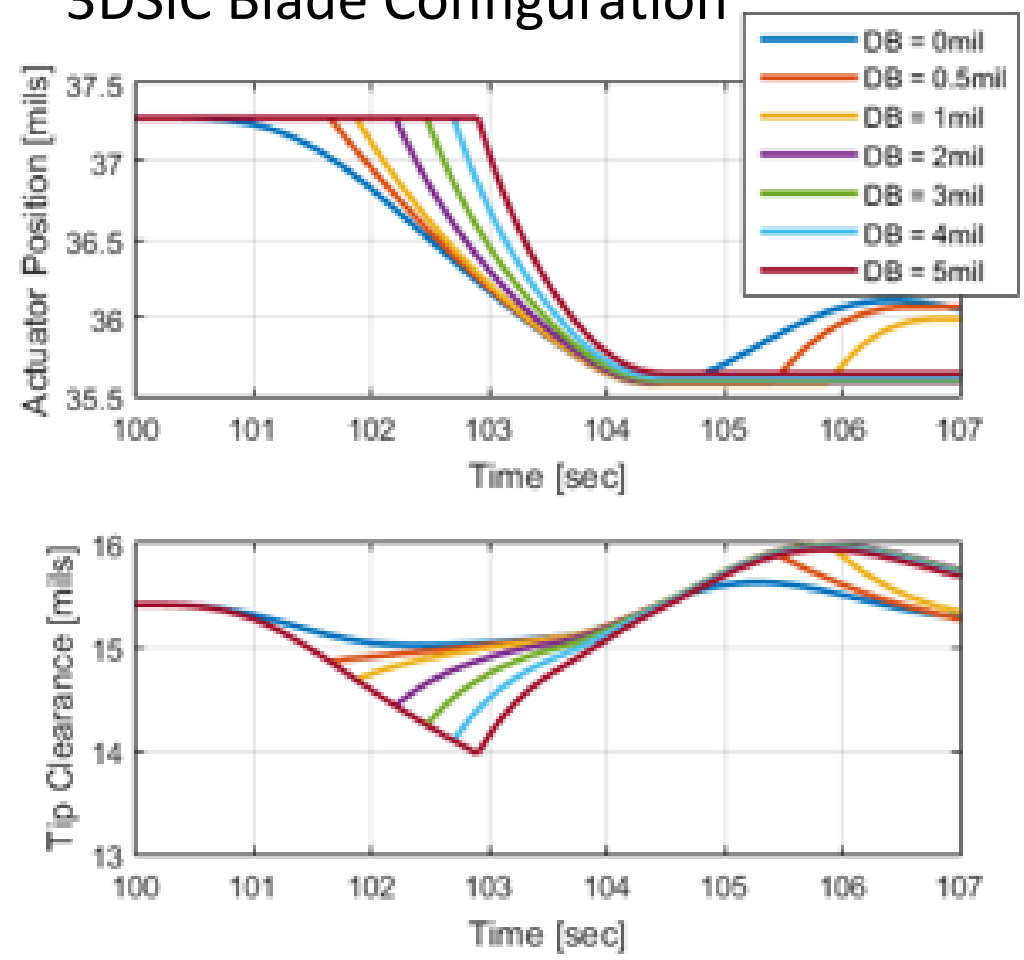

- Recommendations

- Inconel: $\mathrm{DB}<2 \mathrm{mil}$

- 3DSiC DB $<0.5$ mil 


\section{Parametric Actuator Studies}

\section{DSiC Blade Configuration}
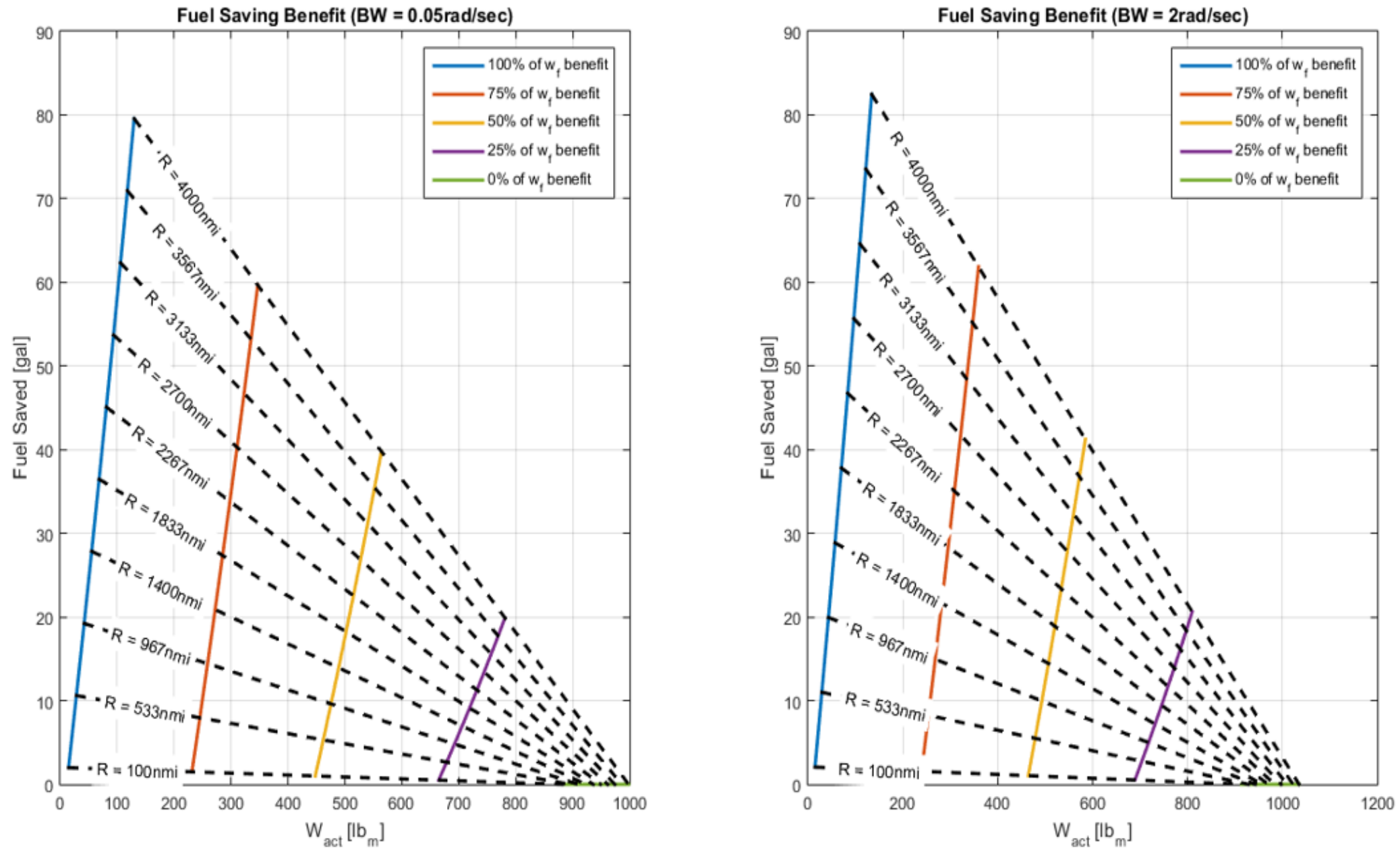


\section{Parametric Actuator Studies}

- Pressure forces are expected to be dominant

- Exact pressure forces may be dependent on design

- Looked at pressure differentials $(\Delta \mathrm{P})$ for 2 basic configurations: (1) modulating the shroud and (2) modulating the shroud/casing assembly in the direction of the net pressure differential across the modulated object
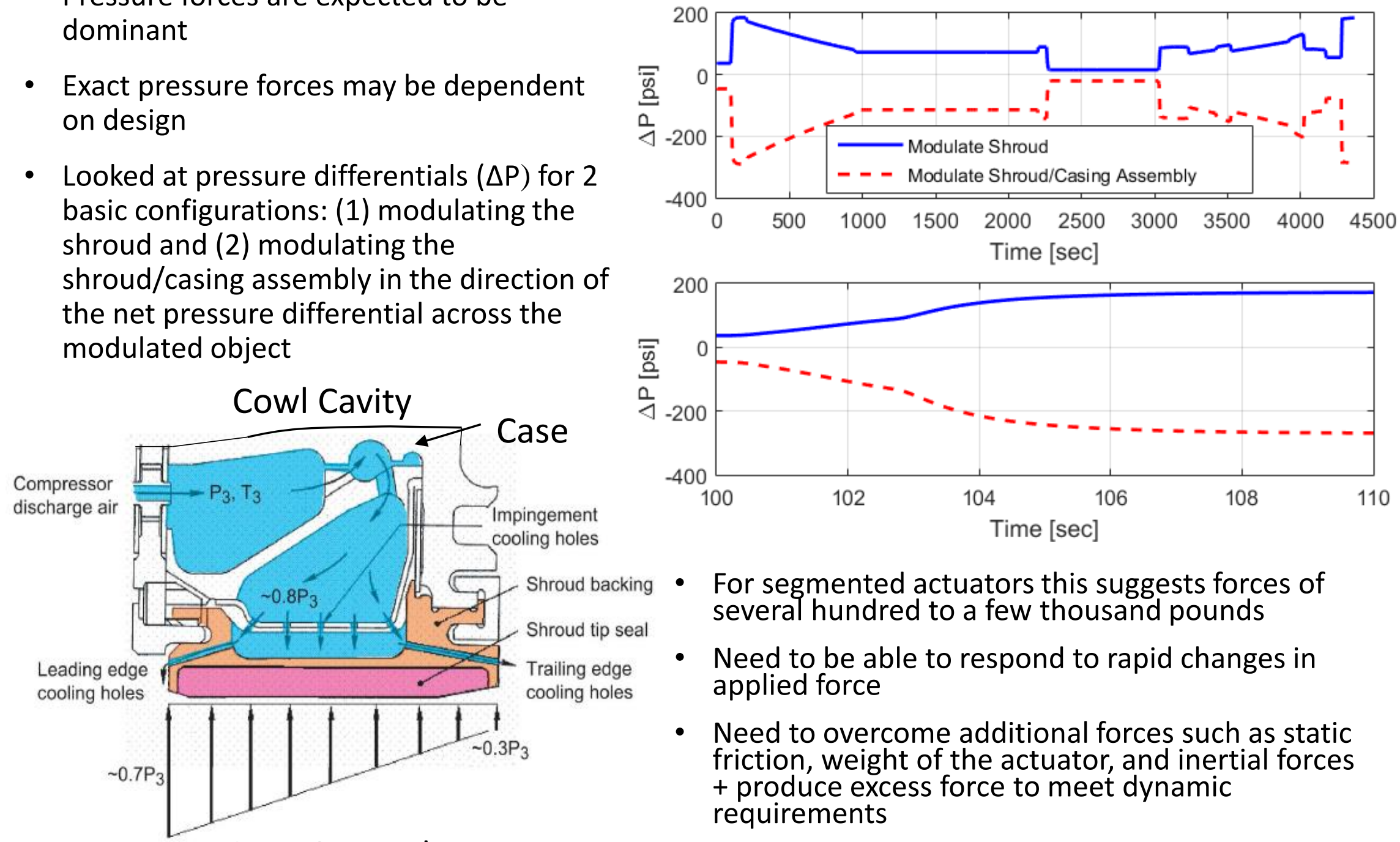

- For segmented actuators this suggests forces of several hundred to a few thousand pounds

- Need to be able to respond to rapid changes in applied force

- Need to overcome additional forces such as static friction, weight of the actuator, and inertial forces + produce excess force to meet dynamic requirements 